\title{
Diseño y desarrollo de un equipo para el control de la calidad en la industria panificadora
}

\author{
A piece of equipment for quality control in the baking \\ industry - design and implementation
}

\author{
Nelson Flórez Ramírez \\ Ingeniero Industrial, Magíster en Educación y Docencia. Docente Investigador del Po- \\ litécnico Jaime Isaza Cadavid. Medellín, Colombia. Contacto: nflorez@elpoli.edu.co \\ Francisco Javier López Correa \\ Ingeniero en Productividad y Calidad, Especialista en Gerencia Integral. Docente inves- \\ tigador del Politécnico Colombiano Jaime Isaza Cadavid. Medellín, Colombia. \\ Contacto: franciscolopez@elpoli.edu.co

\section{Andrea Lucia Flórez Rendón} \\ Ingeniera Industrial, Candidata a Magíster en Desarrollo Sostenible y Medio Ambiente. \\ Docente del Instituto Tecnológico Metropolitano. Medellín, Colombia. \\ Contacto: andreaflorez@itm.edu.co
}

Fecha de recepción: 25 de noviembre de 2011

Clasificación del artículo: Estudio de Caso

Fecha de aceptación: 27 de noviembre de 2012

Financiamiento: Politécnico Colombiano Jaime Isaza Cadavid

Palabras clave: calidad, industria alimentaria, productividad industrial.

Key words: quality, food industry, industrial productivity.

\section{RESUMEN}

La productividad y la calidad han sido protagonistas en la búsqueda de mayores índices de competitividad en las organizaciones, esto permite su crecimiento con apoyo del desarrollo tecnológico. La reducida tecnología a la que acceden las industrias manufactureras y en especial las panificadoras las ha sometido a niveles bajos de competitividad.
Con el objeto de fortalecer el vínculo universidadempresa y de implementar y socializar el conocimiento tecnológico para el crecimiento de las pymes, el Politécnico Colombiano Jaime Isaza Cadavid ejecutó el proyecto "Diseño y Desarrollo de un Equipo para el Control de Calidad en la Industria Panificadora", con el que busca estandarizar y mejorar los niveles de calidad del proceso de fermentación, crítico en la elaboración del pan. 


\section{revisión}

Se recolectaron datos y se realizaron mediciones al producto durante y después del proceso de fermentación. Se analizaron las condiciones del proceso y las variables de mayor incidencia en la fermentación: temperatura y humedad.

Los análisis estadísticos y de calidad demostraron la necesidad de estandarizar el proceso, sus especificaciones y las características de calidad y variables críticas (largo, ancho, altura) del producto por fabricar. Los resultados de los análisis iniciales permitieron identificar potenciales especificaciones y parametrizar con base en estas un equipo para controlar las variables del proceso mediante un escáner que de forma lineal toma imágenes de los productos en el túnel de fermentación, donde se lleva a cabo el proceso, manteniendo las condiciones requeridas, evitando la manipulación del producto y reduciendo en un $50 \%$ el tiempo de fermentación del pan. El equipo permite realizarmediciones precisas y registrar los datos con el fin de evitar la alteración de las condiciones del proceso en sus variables críticas, de esta manera se contribuye así a la fabricación de productos de la más alta calidad.

\begin{abstract}
Productivity and quality have been important in the search for higher levels of competitiveness in organizations. This, together with technological development, has allowed organizations to grow. However, low technological development in manufacturing industries (especially bakeries) has led to low levels of competitiveness in Colombia.
\end{abstract}

"Politécnico Colombiano JIC" implemented a project called "Design and development of a piece of equipment for Quality Control in the breadmaking industry" (also strengthening the bonds between "universities and the industry") in order to implement and socialize technological knowledge for the growth of SMEs. The project was aimed at standardizing and improving the quality levels of fermentation processes (which are critical in bread making). Once data was collected, the product was assessed during and after the process of fermentation. Process conditions were tested together with the variables of greatest incidence in fermentation, namely temperature and humidity.

Statistical analysis and quality tests showed the need for standardization in terms of process design, product specifications and quality characteristics of the product to be manufacture (e.g. length, width and height). The results from initial analysis allowed identifying potential specifications as well as obtaining the parameters of a mechanism for controlling process variables. To accomplish this, pictures of sample products into the fermentation tunnel (where the process takes place while maintaining the required conditions) were taken using a linear scanner. By avoiding the handling of the product, bread fermentation time was reduced by $50 \%$. The mechanism allows making accurate measurements and recording data by preventing alterations in the process conditions for critical variables, which contributes to high-quality manufacture.

\section{INTRODUCCIÓN}

Durante mucho tiempo los productos generados en las grandes factorías fueron entregados a los clientes y consumidores finales bajo los más escasos controles frente a su funcionalidad; en realidad, dichos

controles no estaban orientados al cumplimiento de unos requisitos específicos para los clientes, sino más bien a unas condiciones generales que manifestaba la sociedad respecto a sus necesidades básicas [1]. Fue solo hasta 1910cuandola calidad de los productos empezóa ser importante y los compradores comen- 
zaron a emitir conceptos de rechazo e inconformidad frente a productos que en su apariencia revelaban posibles fallas. De ahí en adelante, hasta 1970, se buscó asegurar la calidad no solo mediante el proceso, sino también en su producto final obtenido[2].

La industria actual ha dejado de ver a la calidad como una ventaja competitiva, convirtiéndose esta en característica inherente a cualquier producto y requisito parapermaneceren el mercado. Sin embargo, el cumplimiento de altos niveles de calidad requiere de inversión y esfuerzos conjuntos dentro de toda organización, lo que definitivamente no es tarea fácil en países en vías de desarrollo que cuentan con limitado acceso a la tecnología y escasos recursos para la inversión en esta [3]. Dicho panorama es el que actualmente viven muchas industrias colombianas y especialmente el sector de alimentos, que ocupa el primer lugar de importancia en los principales indicadores económicos del país [4] y se caracteriza por ser una actividad fundamentalmente de la pequeña y mediana empresa. En particular nos referimos a la industria panificadora, que a pesar de experimentar un crecimiento significativo en el país durante los últimos años, aún carece de sistemas y tecnologías que le permitan obtener niveles de calidad satisfactorios frente a las necesidades del mercado. Esta industria contaba en el año 2008 con 24850 panificadoras registradas a nivel nacional, de las cuales la gran mayoría son Pymes, generando aproximadamente 222715 empleos directos a nivel nacional [4].

La Asociación Colombiana de las Pequeñas yMedianas Empresas (ACOPI) y concretamente el sector de los panaderos de Colombia, buscando mejorar el nivel tecnológico del sector, su productividad y competitividad y con base a un centro de desarrollo tecnológico europeo llamado La Casa del Pan, cuyo objetivo principal es apoyar el desarrollo técnico y tecnológico de este sector, pretenden desarrollar en Colombia un modelo similar que permita fortalecer este sector debido a su notable crecimiento.

Basados en lo anterior y con el fin de reportar beneficios desde la academia hasta la industria, el
Politécnico Colombiano Jaime Isaza Cadavid, en conjunto con la Asociación Colombiana de Pequeñas y Medianas Empresas (ACOPI), plantearon la posibilidad de proponer un proyecto para el diseño y desarrollo de un equipo que permitiera el control de la calidad en este sector con el fin de lograr un crecimiento considerable asociado al mejoramiento sustancial en la calidad de sus productos. De esta manera, el equipo se convirtió en primer aporte al desarrollo de dicho modelo. Este se compone básicamente por un escáner a base de un láser que captura las imágenes al interior del túnel de fermentación, un equipo de cómputo y un software que permiten visualizar las imágenes del producto.

\section{METODOLOGÍA}

La metodología utilizada para el desarrollo del presente proyecto obedece a un modelo de investigación de tipo aplicada orientada a la consecución de un nuevo conocimiento por medio de la aplicación de la información previa al logro del mejoramiento de la productividad y la calidad en la industria panificadora. Para este fin se implementó un equipo tipo escáner [5] que controla las características de calidad más relevantesdel pan como objetivo específico [6]. Se realizaron visitas a la empresa, entrevistas con el personal de las diferentes áreas y mediciones al producto en proceso y producto terminado durante sus diferentes etapas, con el propósito de recolectar información, lo que permitió la definición de las variables de interés por parte de la dirección de la empresa Panificadora Lore S.A., ubicada en el municipio de Medellín.

\section{DESARROLLO}

Luego de que se concretara el compromiso entre el Politécnico Colombiano Jaime Isaza Cadavid y ACOPI, una de las empresas asociadas a esta asociación, Panificadora Lore S.A., fue la organización seleccionada para participar en eldesarrollo de este proyecto, debido su interésen introducir mejoras significativas a su proceso y garantizar la calidad de los productos que hoy se ofrecen a un mercado en notable crecimiento. 
Se parte del estudio del sector panificador en Colombia con el fin de caracterizar y diagnosticar el nivel técnico y tecnológico del que actualmente este dispone en entornos económicos, tecnológicos y organizacionales. Allí se encontró que muy pocas organizaciones están provistas de tecnología adecuada y por el contrario, una gran cantidad de panificadoras tienen procesos productivos muy básicos y artesanales debido a que su conformación obedece principalmente a pequeñas y medianas empresas, que representan un $97 \%$ en las empresas de este sector [4].

La Panificadora Lore S.A., a pesar de disponer deniveles adecuados de tecnología en algunas etapas del proceso, contaba con una serie de dificultades en algunas operaciones de su proceso productivo que generaban altos volúmenes de producción defectuosa, tiempos improductivos, excesiva manipulación de productos en proceso y pocos controles que le permitieran identificar y controlar las causas de dichos problemas.
Hecha la descripción del proceso productivo a través de un diagrama de flujo del proceso (figura 1), se realizó un análisis del sistema de producción, lo que permitió identificar algunas de las causas más significativas de los problemas detectados, arrojando como principal resultado la identificación del proceso de fermentación como el más crítico del proceso productivo. En la fermentación se definen las más importantes características del producto terminado (volumen, sabor, consistencia, textura y durabilidad), mediante el control de las variables temperatura y humedad. Es además la operación de mayor tiempo en el proceso (de 3 a 3,5 horas actualmente), constituye el cuello de botella de este y adicionalmente genera mayor producción defectuosa [7]. Sumado a lo anterior, la empresa no dispone de información confiable para tomar acciones correctivas, preventivas y de mejoramiento ni definir estándares de calidad y tiempo para este proceso y poder ejercer controles que garanticen un buen nivel de calidad, una mayor eficiencia y productividad. 


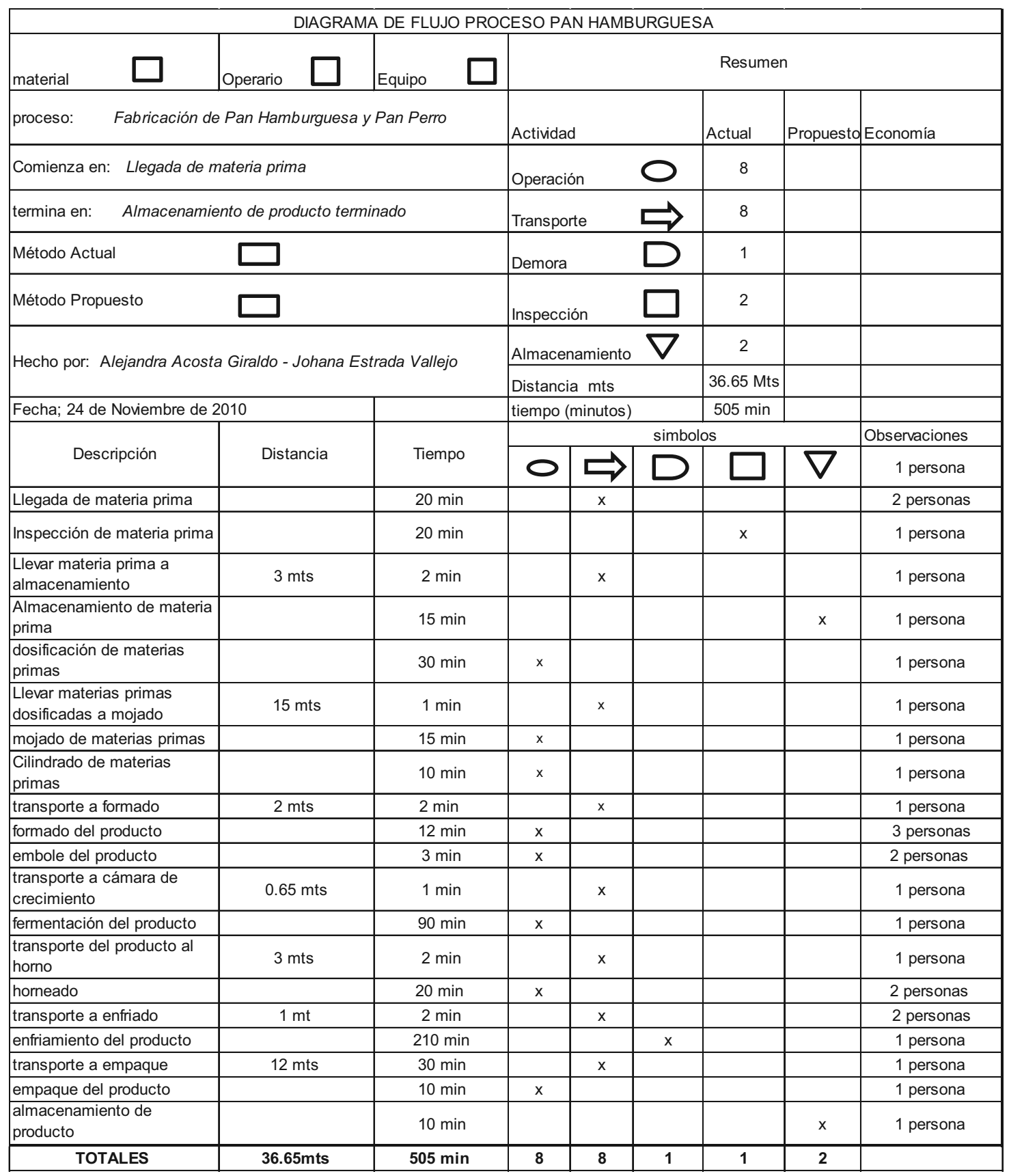

Figura 1. Diagrama de flujo del proceso "pan hamburguesa"

Fuente: elaboración propia.

Los métodos de inspección automatizados verifican la calidad solamente en unas cuantas muestras en determinado periodo de tiempo para cada uno de los productos elaborados. Aunque los detectores de metal, equipos de control y chequeadores de peso proveen el último chequeo de los productos antes de que el producto salga de la planta, se necesitan otros métodos que se anticipen en el proceso[8], es decir, 
que se ejecuten antes de agregarle más valor al producto o antes del empacado. En años recientes, los procesadores han empezado a utilizar los sistemas de visión ópticos en línea y las máquinas de visión para lograr mejoras en la calidad [9], suministrando información para el diseño de las acciones requeridas en lo relacionado con la calidad del producto. Las visualizaciones gráficas en tiempo real y las alarmas alertan a los operadores para que efectúen cambios mucho antes de que las diferenciassean visibles para el ojo humano [10]. Eso significa ajustar las condiciones de la línea antes de que el producto semi elaborado o final,esté fuera de especificación, lo que permite a un operador trabajar múltiples líneas extendiendo el costo de mano de obra más hacia el rendimiento. La conformidad de las especificaciones puede ser considerada a través de los diferentes turnos, reduciendo la variación, mejorando el nivel de calidad y ofreciendo una manera no incriminatoria de resolver problemas [11].

Luego de un análisis de la importancia de esta etapa del proceso y la urgencia de normalizar su desarrollo en aras de mejorar la calidad del producto, se concluyó la necesidad de implementar a esta etapa un sistema que permitiera controlar y monitorear continuamente el desarrollo del producto en la fermentación, a fin de disminuir la manipulación del producto, estandarizar las especificaciones de calidad de cada una de las características y por medio de estas definir los parámetros mediante los cuales se puede garantizar que el producto cumple las condiciones requeridas.

Conocidas las necesidades de la organización y después de identificar los requerimientos técnicos para el desarrollo de un sistema adecuado, se decidió diseñar un equipo a base de un sistema de visión utilizado en lacaptura de imágenes y su posterior procesamiento, análisis e interpretación para dar una retroalimentación a tiempo [12], por lo que inicialmente se pensó en un sistema en dos dimensiones que utiliza una imagen plana para monitorear la forma y apariencia visual del producto controlado. Sin embargo, debido a la importancia del proceso analizado, se definió la utilización de un equipo en tres dimensiones, con características superiores al que previamente se había pensado debido a la posibilidad que ofrece de observar el producto en proceso desde uno o más ángulos y la determinación de la altura de cada punto del objeto, analizado todo mediante la utilización de una fuente de luz estructurada, razones por las cuales un equipo de visión tridimensional se convierte en una herramienta eficaz para controlar procesos de diferentes industrias y características de las mismas [13], que para el caso particular de la Panificadora Lore S.A., permiten mantener un registro del comportamiento del producto dentro del proceso de fermentación, dando a este un tiempo definido y garantizando la conformidad del producto resultante.

El equipo diseñado es un sistema de visión en tres dimensiones con movimiento lineal que se compone básicamente por un escáner a base de un láser que captura las imágenes al interior del túnel de fermentación, un equipo de cómputo y un software que permiten visualizar las imágenes del producto escaneado, analizar sus dimensiones y almacenar la información obtenida de las diferentes muestras a realizar. La función del equipo es fundamentalmente controlar las dimensiones del producto en función del largo, ancho y altura. De acuerdo con una frecuencia definida o a la activación manual del sistema, el escáner capta las imágenes del producto al interior del túnel de fermentación. Esta imagen se proyecta en el computador, donde es posible analizar la forma del producto y conocer el volumen que registra en cualquier momento del proceso, de manera que una vez definidas las especificaciones de volumen de este, el equipo es parametrizado para que en el momento en que el volumen del producto se encuentre dentro de este rango, el sistema emita una señal representada por luces y sonido que da a conocer al personal del área que el producto ya cumplió el proceso de fermentación y que puede darse paso a la siguiente etapa.

Una vez instalado el equipo, se desarrollaron inicialmente muestreos aleatorios manuales (tablas 1 y 2) 
al producto a fermentar, antes, durante y después de dicho proceso, utilizando instrumentos de medición, con el fin de establecer unos parámetros iniciales para poner en funcionamiento el equipo. Posterior a este muestreo, se empleó el equipo diseñado para tomar diferentes muestras del volumen registrado por los productos a analizar que fueron el "pan perro" y el "pan hamburguesa", los productos de mayor rotación en el mercado de la panificadora. Con base en estas mediciones y pruebas realizadas, se definieron las especificaciones de volumen para cada uno de los productos, las que actualmente permiten alertar al operario del proceso en qué momento debe retirar el producto del proceso de fermentación.

Tabla 1. Ejemplo del muestreo manual pan hamburguesa y pan perro.

\begin{tabular}{|c|c|c|c|c|}
\hline \multicolumn{2}{|c|}{ PRODUCTO: Pan hamburguesa } & \multicolumn{3}{|c|}{ PROCESO: Formado } \\
\hline FECHA & LARGO & ANCHO & ALTURA & VOLUMEN \\
\hline 07/10/2010 & 9,20 & 9,20 & 2,30 & 194,67 \\
\hline 07/10/2010 & 9,20 & 9,20 & 1,50 & 126,96 \\
\hline $07 / 10 / 2010$ & 10,00 & 11,00 & 1,50 & 165,00 \\
\hline $07 / 10 / 2010$ & 10,20 & 10,00 & 1,90 & 193,80 \\
\hline $07 / 10 / 2010$ & 8,00 & 8,00 & 2,00 & 128,00 \\
\hline $07 / 10 / 2010$ & 7,50 & 7,50 & 2,30 & 129,38 \\
\hline $07 / 10 / 2010$ & 8,50 & 8,50 & 2,00 & 144,50 \\
\hline $07 / 10 / 2010$ & 9,00 & 9,00 & 2,00 & 162,00 \\
\hline 07/10/2010 & 8,90 & 9,00 & 2,30 & 184,23 \\
\hline PROMEDIO & 8,94 & 9,04 & 1,98 & 158,73 \\
\hline MÁXIMO & 10,2 & 11 & 2,3 & 194,67 \\
\hline MÍNIMO & 7,5 & 7,5 & 1,5 & 126,96 \\
\hline
\end{tabular}

Fuente: elaboración propia. 


\section{revisión}

Tabla 2. Mediciones con el equipo

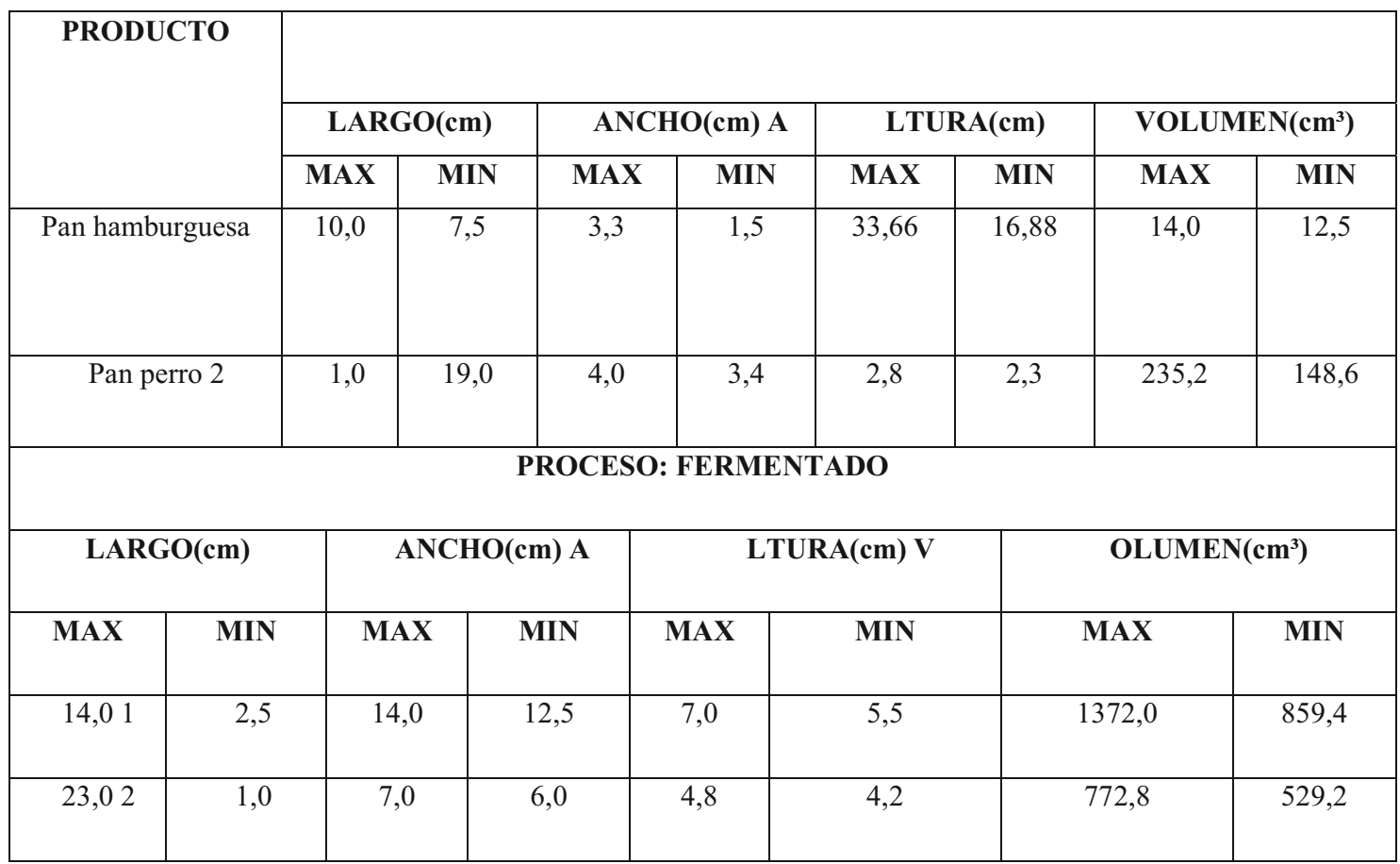

Fuente: elaboración propia.

\section{RESULTADOS}

Como resultado de gran importancia en el proyecto, se definieron los valores a recomendar como especificaciones para las dimensiones del producto, tanto en el proceso de formado como en el proceso de fermentación, que fue el de mayor interés para el proyecto ejecutado. Por medio de estas especifi- caciones, se definieron los parámetros del equipo que permiten que este alerte al personal cuando el producto ha completado el proceso, dichos resultados especifican los valores máximos y mínimos que deben registrarse en las diferentes dimensiones de los productos (tablas 3 y 4 ).

Tabla 3. Proceso: Formado.

\begin{tabular}{|c|c|c|c|c|c|c|c|c|}
\hline \multirow{2}{*}{ PRODUCTO } & \multicolumn{9}{|c|}{ PROCESO: Formado } \\
\cline { 2 - 10 } & \multicolumn{2}{|c|}{ LARGO $(\mathrm{cm})$} & \multicolumn{2}{|c|}{ ANCHO $(\mathrm{cm})$} & \multicolumn{2}{|c|}{ ALTURA $(\mathrm{cm})$} & \multicolumn{2}{c|}{ VOLUMEN $\left(\mathrm{cm}^{3}\right)$} \\
\cline { 2 - 9 } & MAX & MIN & MAX & MIN & MAX & MIN & MAX & MIN \\
\hline \multirow{2}{*}{ Pan hamburguesa } & 10,0 & 7,5 & 3,3 & 1,5 & 33,66 & 16,88 & 14,0 & 12,5 \\
\hline Pan perro & 21,0 & 19,0 & 4,0 & 3,4 & 2,8 & 2,3 & 235,2 & 148,6 \\
\hline
\end{tabular}

Fuente: elaboración propia. 
Tabla 4. Proceso: Fermentado

\begin{tabular}{|c|c|c|c|c|c|c|c|c|}
\hline \multirow{2}{*}{ PRODUCTO } & \multicolumn{9}{|c|}{ PROCESO: Fermentado } \\
\cline { 2 - 9 } & \multicolumn{2}{|c|}{ LARGO $(\mathrm{cm})$} & \multicolumn{2}{c|}{ ANCHO $(\mathrm{cm})$} & \multicolumn{2}{c|}{ ALTURA $(\mathrm{cm})$} & \multicolumn{2}{c|}{ VOLUMEN $\left(\mathrm{cm}^{3}\right)$} \\
\cline { 2 - 9 } & MAX & MIN & MAX & MIN & MAX & MIN & MAX & MIN \\
\hline Pan hamburguesa & 14,0 & 12,5 & 14,0 & 12,5 & 7,0 & 5,5 & 1372,0 & 859,4 \\
\hline Pan perro & 23,0 & 21,0 & 7,0 & 6,0 & 4,8 & 4,2 & 772,8 & 529,2 \\
\hline
\end{tabular}

Fuente: elaboración propia.

Conforme a los parámetros enunciados anteriormente fue posible definir las especificaciones a manejar por el equipo instalado. De tal manera, cuando el producto en fermentación, bien sea pan perro o pan hamburguesa, está dentro del rango de volumen especificado, el equipo emite una alerta que informa al operador que el proceso de fermentación ha finalizado y que el producto se encuentra listo para ser horneado. Por esto, uno de los resultados más importantes es la eliminación de la manipulación del producto en proceso y la alteración de las condiciones de humedad y temperatura del túnel de fermentación, ya que no es necesario que durante el proceso el operador saque el producto para verificar su estado ni que por cuenta de abrir la puerta del túnel altere las condiciones de este.

Tabla 5. Cuadro comparativo entre los dos sistemas.

\begin{tabular}{|c|c|}
\hline Sistema 2D & Sistema 3D \\
\hline $\begin{array}{l}\text { Toma mediciones solo en dos dimensiones que pueden ser } \\
\text { ancho y largo }\end{array}$ & $\begin{array}{l}\text { Toma mediciones en tres dimensiones: } \\
\text { largo, ancho, alto }\end{array}$ \\
\hline $\begin{array}{l}\text { Las mediciones de distancia corresponden a la } \\
\text { interpretación de los pixeles de la imagen }\end{array}$ & $\begin{array}{l}\text { La medición de distancia es una combinación entre la inter- } \\
\text { pretación de los pixeles y la medición por triangulación }\end{array}$ \\
\hline $\begin{array}{l}\text { En el sistema 2D toma las mediciones un una } \\
\text { sola imagen }\end{array}$ & $\begin{array}{l}\text { En el sistema 3D se debe de hacer un barrido de } \\
\text { imágenes y luego concatenarlas para formar una imagen }\end{array}$ \\
\hline Se analiza el espacio en un vector & Se analiza el espacio en una matriz \\
\hline La resolución solo depende del sensor de la cámara & $\begin{array}{c}\text { La resolución depende del sensor de la cámara y de los } \\
\text { motores lineales }\end{array}$ \\
\hline $\begin{array}{l}\text { El sistema requiere un sistema de iluminación complejo y } \\
\text { costoso }\end{array}$ & $\begin{array}{c}\text { El sistema no requiere iluminación, pero es susceptible a } \\
\text { cambios de iluminación ambiental }\end{array}$ \\
\hline $\begin{array}{c}\text { Es un sistema es rápido y requiere poca capacidad } \\
\text { computacional }\end{array}$ & $\begin{array}{l}\text { Es un sistema lento y requiere alta capacidad } \\
\text { computacional }\end{array}$ \\
\hline $\begin{array}{l}\text { Se necesita realizar cálculos adicionales para hallar una co- } \\
\text { rrelación entre el área y el volumen del pan }\end{array}$ & $\begin{array}{l}\text { El sistema permite medir directamente el volumen del pan } \\
\text { siendo más preciso en las medidas }\end{array}$ \\
\hline El consumo de potencia depende del sistema de iluminación & El consumo de potencia depende de los motores lineales \\
\hline $\begin{array}{l}\text { El costo de implantación depende de los accesorios y siste- } \\
\text { mas de iluminación }\end{array}$ & $\begin{array}{l}\text { El costo de implementación depende de los sistemas láser } \\
\text { y de los motores lineales y su control }\end{array}$ \\
\hline Precisión de $\pm 0,8 \mathrm{~mm}$ & Precisión de $\pm 0,01 \mathrm{~mm}$ \\
\hline Costo aproximado de implementación: $\$ 20000000$ pesos & Costo aproximado de implementación: $\$ 110000000$ pesos \\
\hline
\end{tabular}

Fuente: elaboración propia. 


\section{revisión}

Debido a la automatización del proceso de fermentado, el tiempo de esta etapa del proceso pudo reducirse, resultado obtenido por medio de un estudio de tiempos realizado al proceso de fermentación antes y después de instalado el equipo, como se explica a continuación.

\subsection{Disminución del tiempo de inspección en proceso de leudación}

En el proceso de leudación, se debe cumplir un tiempo mínimo de acción del componente del pan que genera el crecimiento, como lo es lalevadura. Adicionalmente se deben cumplir las especificaciones de temperatura $\left(28^{\circ}\right)$ y de humedad relativa de $(70 \%)$, estas son las que generan mayor o menor proceso de la levadura. Con el diseño y puesta en marcha del equipo para controlar la calidad del pan se requiere homogeneidad en este proceso, así se disminuye el tiempo de fermentación. Se puede verificar que la disminución de tiempo está entre sesenta (60) minutos, una (1) hora y setenta y cinco (75) minutos, una hora y uncuarto $\left(1 \frac{1}{4}\right)$, de acuerdo con los datos obtenidos en el estudio de tiempos.

\subsection{Disminución del tiempo de inspección en proceso de fermentación}

En el proceso de fermentación, se debe cumplir un tiempo mínimo de acción del componente del pan que genera el crecimiento, como lo es la levadura. Adicional a este tiempo se deben cumplir las especificaciones de temperatura $\left(28^{\circ}\right)$ y de humedad relativa de (70), estas son las que generan mayor o menor proceso de la levadura. Con el diseño y puesta en marcha del equipo para controlar la calidad del pan se requiere homogeneidad en este proceso, así se disminuye el tiempo de fermentación. Se puede verificar que la disminución de tiempo está entre sesenta (60) minutos, una (1) hora y setenta y cinco (75) minutos, y una hora y un cuarto $\left(1 \frac{1}{4}\right)$.

\subsection{Disminución de la manipulación del producto}

Luego de la fermentación, el proceso siguiente es el horneo. Se podría llevar directamente de las cabinas de fermentación hacia los hornos, si se hubieran respetado las características de temperatura y humedad relativa. Como el cumplimiento no es apto, se hace necesaria una revisión de cada una de las bandejas con producto, para saber cuáles tienen el volumen y tamaño ideal para llevar a hornear.

Este proceso de manipulación de bandejas lleva aproximadamente 12,39 segundos por bandeja, además se tiene que si trabajan 30 bandejas por escabiladero (carro), se producen en forma general entre sesenta y setenta carros por día, lo que significa que se trabajan aproximadamente entre 1800 y 2100 bandejas por día, esto da un tiempo total por día entre 371,7 y 433,65 minutos perdidos en revisión por día, es decir, entre 6,20 y 7,23 horas.

Esto se mejorará cuando se adecuen las cabinas y se homogenicen las condiciones de temperatura y humedad relativa. El computador del equipo que registra los volúmenes del pan, suministrará oportunamente los valores de la especificación del volumen del producto, eliminando el tiempo de revisión, la pérdida de temperatura y la manipulación.

\subsection{Mejorade la eficiencia, eficacia y productividad}

Estas disminuciones de tiempos inciden en la mejora de eficiencia, eficacia y la productividad.

\subsection{Disminución de los costos de producto terminado}

Al disminuir los tiempos de leudación o fermentación y de manipulación o revisión de productos para saber si cumplen con la especificación de volumen y seguir el proceso de horneado, los costos del producto terminado disminuyen, toda vez que el tiempo de mano de obra directa es menor al utilizar el equipo de calidad para el pan. 
En las tablas 6, 7 y 8 se observa cuánto se ahorrará una organización por implementar la mejora de su proceso con el equipo de control de calidad para el proceso de fermentación.

Tabla 6. Proceso: Ahorro de la organización.

\begin{tabular}{|c|c|c|c|c|c|}
\hline $\begin{array}{c}\text { SMLMV } \\
(2012)\end{array}$ & $\begin{array}{c}\text { FACTOR } \\
\text { PRESTACIONAL }\end{array}$ & SUBTOTAL & $\begin{array}{c}\text { SUBSIDIO DE } \\
\text { TRANSPORTE * }\end{array}$ & COSTO MES & $\begin{array}{c}\text { COSTO POR } \\
\text { HORA }\end{array}$ \\
\hline$\$ 566700$ & $1,5340 \%$ & $\$ 869317,80$ & $\$ 67800$ & $\$ 937117,80$ & $\$ 3904,66$ \\
\hline
\end{tabular}

Fuente: elaboración propia.

Para encontrar el costo ahorrado por día, según los que en total suman entre 7,20 y 8,38 horas, tenemos tiempos perdidos en fermentación y en revisión, lo siguiente:

Tabla 7. Ahorro mes mínimo.

\begin{tabular}{|c|c|c|c|c|c|c|c|c|}
\hline \$ Hora & $\begin{array}{c}\text { Horas } \\
\text { día }\end{array}$ & \$Ahorro día & $\begin{array}{c}\text { Días } \\
\text { semana }\end{array}$ & $\begin{array}{c}\text { \$ Ahorro se- } \\
\text { mana }\end{array}$ & $\begin{array}{c}\text { Semanas } \\
\text { mes }\end{array}$ & $\begin{array}{c}\text { \$ Ahorro } \\
\text { mes }\end{array}$ & $\begin{array}{c}\text { Meses } \\
\text { año }\end{array}$ & $\begin{array}{c}\text { \$ Ahorro } \\
\text { año } \\
\$ 3904,66\end{array}$ \\
7,25 & $\$ 28308,79$ & 6 & $\$ 169852,7$ & 4,3328 & $\$ 735937.8$ & 12 & $\$ 8831253,8$ \\
\hline
\end{tabular}

Fuente: elaboración propia.

Tabla 8. Ahorro máximo del mes.

\begin{tabular}{|c|c|c|c|c|c|c|c|c|}
\hline \$ Hora & $\begin{array}{c}\text { Horas } \\
\text { Día }\end{array}$ & $\begin{array}{c}\text { \$ Ahorro } \\
\text { Día }\end{array}$ & $\begin{array}{c}\text { Días Se- } \\
\text { mana }\end{array}$ & $\begin{array}{c}\text { \$ Ahorro } \\
\text { Semana }\end{array}$ & $\begin{array}{c}\text { Semanas } \\
\text { Mes }\end{array}$ & \$ Ahorro Mes & $\begin{array}{c}\text { Meses Año } \\
\text { A Ahorro } \\
\text { Año }\end{array}$ \\
\hline$\$ 3904,6$ & 8,45 & $\$ 32994,3$ & 6 & $\$ 197933,2$ & 4,3328 & $\$ 857748,21$ & 12 & $\$ 10292978$ \\
\hline
\end{tabular}

Fuente: elaboración propia.

Dentro de la empresa Panificadora Lore S.A. se presentaban problemas de homogeneidad en las condiciones de humedad y temperatura del túnel de fermentación, esto ocasionado no solo por la manipulación continua del producto sino también por el estado del túnel como tal, que no garantizaba condiciones de hermeticidad y que debido al sistema empleado para la generación de temperatura no garantizaba que esta pudiera distribuirse uniformemente dentro del área requerida. A partir de esta necesidad y la de garantizar el óptimo aprovechamiento del equipo diseñado, se sugirió a la empresa realizar adecuaciones al túnel de fermentación, lo que se evidencia en los siguientes registros fotográficos. 

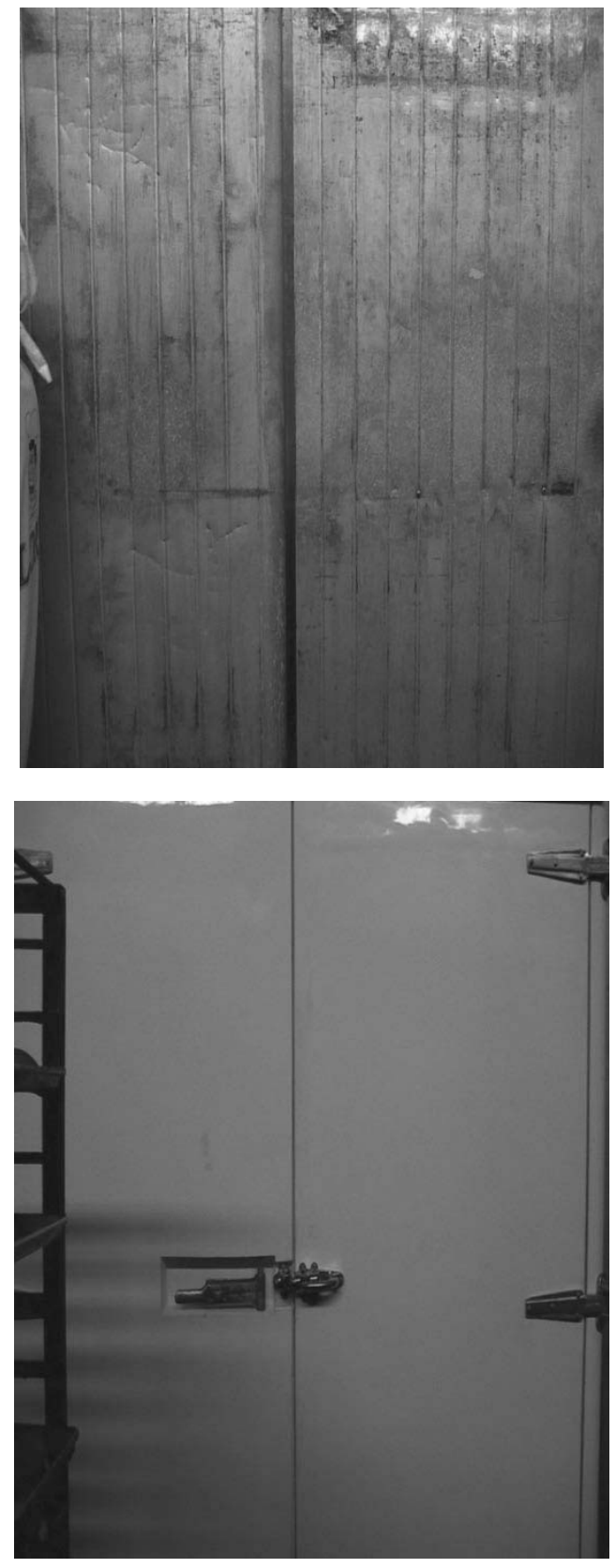

Figura 2. Adecuaciones túnel de fermentación. Fuente: elaboración propia.

Se diseñaron dentro de la empresa Panificadora Lore S.A. nuevos moldes para el proceso de fermentado, los cuales anteriormente eran superficies de aluminio lisas donde el producto se disponía para entrar al túnel de fermentación. Sin embargo, dentro del desarrollo del producto se evidenció la necesidad —después de conocer las especificaciones del volumen en el proceso de fermentado- de construir moldes ya no con una superficie lisa sino con concavidades específicas para contener el producto, puesto que de esta manera se evita que en el proceso unas unidades se unan a otras o no cuenten con espacio suficiente para su crecimiento.

\section{CONCLUSIONES}

El equipo diseñado y desarrollado para el control de la calidad en la industria panificadora, permite garantizar a través de la verificación del volumen la uniformidad en el producto y mejorar el nivel de calidad.

El empleo de este equipo permite además controlar algunas otras variables del proceso de fermentación como peso, forma, tamaño, etc., garantizando mejorar los tiempos de producción, eficiencia, eficacia y productividad.

Para obtener una mayor eficiencia en el equipo es necesario homogenizar el proceso de fermentación, reubicando las ollas de vapor como se recomendó.

Durante el proceso de investigación, se validó la viabilidad técnica y conveniencia del prototipo para realizar control de calidad; se pudieron valorar algunos beneficios económicos del proyecto.

Se extrae que el proceso tendrá mejoras, siempre y cuando se acondicionen las cabinas de fermentación, pues con ello se garantizará la homogeneidad de las variables a controlar como lo son la temperatura y la humedad relativa. Significa esto que los tiempos de trabajo se disminuirán en buena proporción.

Con las cabinas de leudación optimizadas, se obtendrán ahorros entre 735937,82 \$/mes y $857748,21 \$ /$ mes. También se puede mostrar como ahorro anual, lo siguiente: entre 8831253,80\$/año y 10292978\$/año.

Se definieron los parámetros de las variables, largo, ancho y altura del pan perro y pan hamburguesa y 
sus respectivas tolerancias, con base a los datos suministrados por el equipo, y se evidenció la diferencia entre el control manual y el control con el equipo, mejorando la precisión del proceso y de los productos.

\section{FINANCIAMIENTO}

Esta investigación es científica y tecnológica, su desarrollo fue avalado y financiado por el Politécnico Jaime Isaza Cadavid y la empresa Panificadora Lore S.A.

\section{AGRADECIMIENTOS}

AAlejandra Acosta y Johana Estrada, por la elaboración de la figura 1 (Diagrama de flujo del proceso "pan hamburguesa"), y a Francisco J. López C. y Nelson Flórez Ramírez, por la elaboración de la tabla 5 (Cuadro comparativo entre los dos sistemas).

\section{REFERENCIAS}

[1] Mettler-Toledo Ltd, Revista Mettler Toledo, 10 octubre 2008. [En línea]. Available: http:// es.mt.com/mt_ext_files/Editorial/Generic/6/ Product_Inspection_Bakery_News_2_Editorial-Generic_11. [Último acceso: 20 febrero 2009].

[2] H. Mariño Navarrete, Planeación estratégica de la calidad total, Bogota: Tercer Mundo, 1996.

[3] H. Lorraine, Bakery andsnaks, 31 octubre 2005. [En línea]. Available: http://www.bakeryandsnacks.com/news/ng.asp?id=63611ccfra-calibre-control-internati-quality-control. [Último acceso: 10 agosto 2011].

[4] Servicio Nacional de Aprendizaje - SENA, Caracterización ocupacional actualización industria de la panificación y la repostería en Colombia, Servicio Nacional de Aprendizaje - SENA, Colombia, 2006.

[5] C. Teutsch, Model-based Analysis and Evaluation of Point Sets from Optical 3D Laser Scanners, Shaker: Verlag, 2007.

[6] M. Levoy, K. Pulli, S. Runsinkiewicz, D. Koller, L. Pereira, M. Ginzton, S. Ander- son, J. Davis, J. Ginsberg, J. Shade and D. Fulk, "The digital Michelangelo project: 3D scanning of large statues", SIGGRAPH ' 00 Proceedings of the 27th Annual Conference on Computer Graphics and Interactive Techniques, pp. 131-144, 2000.

[7] OIT (Oficina Internacional del Trabajo), Introducción al estudio del trabajo, México: Limusa, 2006.

[8] F. Blais, M. Picard and G. Godin, "3DPVT '04 Proceedings of the 3D Data Processing, Visualization, and Transmission, 2nd International Symposium", IEEE Computer Society Washington, pp. 422-429, 2004.

[9] K. lkeuchi, "Modeling from Reality", Third International Conference on 3-D Digital Imaging and Modeling, pp. 117-124, 2001.

[10] J. P. Lavelle, S. R. Schuetand D. J. Schuet, "High Speed 3D Scanner with Real-Time 3D Processing", IEEE IST International Workshop on Imaging Systems and Techniques, pp. 13-17, 2004.

[11] A. Lerner, Estrategias y abordajes metodológicos empleados para incrementar la 


\section{revisión}

mejora continua en las organizaciones, 17 septiembre 2010. [En línea]. Available: http://www.degerencia.com/articulo/ estrategias-y-abordajes-para-incrementarla-mejora-continua-en-las-organizaciones. [Últimoacceso: 1 febrero 2011].

[12] S. Zhang and P.S. Huang, "High-resolution, real-time three-dimensional shape measurement",Optical Engineering, pp. $1-8,2006$.
[13] Q. Chen and T. Wada, "A Light Modulation/Demodulation Method for Real-Time 3D Imaging",Fifth International Conference on 3-D Digital Imaging and Modeling (3DIM’05), pp. 15-21, 2005.

[14] B. Curless , "Computer Graphics", $A C M$ SIGGRAPH, pp. 38-41, 2000. 